\title{
Hindcasting Violent Events in Colombia Using Internet Data
}

\author{
ASHLYNN R. DAUGHTON and SARA Y. DEL VALLE, Los Alamos National Laboratory \\ CHRYSM WATSON ROSS, Los Alamos National Laboratory and University of New Mexico \\ GEOFFREY FAIRCHILD, Los Alamos National Laboratory
}

\begin{abstract}
Colombia experienced a decades-long civil war between the government and many left-wing guerrilla groups. It was marked by violence, kidnappings, and large quantities of human displacement. Monitoring and forecasting civil wars are important to mitigate their potential impact but require access to ground truth data. We examine the use of Internet data streams, namely Google search queries, tweets related to politics, and traditional news sources to retrospectively forecast (i.e., hindcast) statebased armed violence in Colombia. We compare the results of statistical models using three combinations of these features to evaluate the predictive capabilities of each data stream. Our results show that the combination of Internet and traditional news data models perform most consistently, although Internet-only is surprisingly promising. Overall, we are able to produce highquality models hindcasting the presence or absence of state-based armed violence in Colombia up to 6 months in advance. These results support the use of exogenous data streams to forecast evolving situations around the globe.
\end{abstract}

CCS Concepts: • Applied computing $\rightarrow$ Computing in government; $\bullet$ Human-centered computing $\rightarrow$ Empirical studies in collaborative and social computing; • Computing methodologies $\rightarrow$ Topic modeling;

Additional Key Words and Phrases: Social media, Internet data, predictive analyses, political unrest, Colombia

ACM Reference format:

Ashlynn R. Daughton, Sara Y. Del Valle, Chrysm Watson Ross, and Geoffrey Fairchild. 2021. Hindcasting Violent Events in Colombia Using Internet Data. Digit. Gov.: Res. Pract. 2, 3, Article 28 (July 2021), 14 pages.

https://doi.org/10.1145/3462211

\section{INTRODUCTION}

Recent years have seen an especially high incidence of violent conflicts, even in countries that have historically been considered stable [9]. Concurrently, there has been renewed attention focused on the ability to prevent conflict, often by predicting it $[9,21]$. In fact, the World Bank's recent Pathways For Peace report specifically notes the shift "toward preventing conflict sustainably, inclusively, and collectively" [9].

Separately, Internet data have been used in many applications to better understand a range of phenomenon including identification of cases of drug-related adverse events on social media platforms [10], predicting successful romantic relationships [44], and examining the relationship between violent events and hate speech patterns

Research support was provided by the UC Office of the President through its UC National Laboratory Fees Research Program (award LFR18-547591) and the U.S. Department of Energy through the Los Alamos National Laboratory. Los Alamos National Laboratory is operated by Triad National Security LLC for the National Nuclear Security Administration of the U.S. Department of Energy (Contract no. 89233218CNA000001). LA-UR-20-20498.

Authors' addresses: A. R. Daughton, S. Y. Del Valle, and G. Fairchild, Los Alamos National Laboratory, Bikini Atoll Road, SM-30, P.O. Box 1663, Los Alamos, NM 87547; emails: adaughton@lanl.gov, sdelvall@lanl.gov, chrysm@lanl.go, gfairchild@lanl.gov; C. W. Ross, Los Alamos National Laboratory, Los Alamos, NM 87547, and University of New Mexico, Department of Computer Science, University of New Mexico, 1901 Redondo S Dr, Albuquerque, NM 87106.

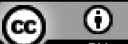

This work is licensed under a Creative Commons Attribution International 4.0 License.

(C) 2021 Copyright held by the owner/author(s).

2639-0175/2021/07-ART28

https://doi.org/10.1145/3462211 
in online communities [32]. In the disease forecasting realm, Internet data have been found to be predictive of a wide number of illness and conditions ranging from depression $[14,15]$ to infectious diseases (e.g., [36]).

This work seeks to evaluate the use of Internet data to predict violent events. We use a high-quality ground truth violence dataset from the Uppsala Conflict Data Program (UCDP) [18, 35, 45], and features derived from Google search queries, Twitter, and the event-based dataset, the Integrated Crisis Early Warning System (ICEWS) [11], to evaluate the predictability of violent events in Colombia. We focus on Colombia as our test case because of the history of violent events stemming from a long and complicated civil war.

Although previous work has used Internet data for forecasting events (e.g., protests, civil unrest, or violence), this is the first study, to our knowledge, that evaluates the predictive capability of Internet data in conjunction with traditional data streams for violent events at a month time resolution. Specifically, we focus on answering the following research questions:

(1) $R Q$ 1: In Colombia, can violent events be hindcast using Internet data. What are the optimal model types (Ridge, Lasso, or ElasticNet), when does a particular model become stale, and what is the maximum forecast horizon (i.e., how far in advance can a violent event be predicted)?

(2) $R Q$ 2: Do these models benefit from a variety of data streams? In particular, do models with Internet data outperform those using the traditional event-based datasets?

Although the operational usefulness of this work would be to forecast violent events in real time, all of the available data to develop and test models exists retrospectively. Thus, throughout this article, we specifically use retrospective forecasting, or hindcasting.

\section{RELATED WORK}

\subsection{Colombian Peace Process}

Violence in Colombia has a long and complex history dating back to its independence from Spain in the early 1800s [24]. Colombia has experienced multiple civil conflicts. The first started in the 1940s and laid the groundwork for the issues in the more recent decades-long civil war that began in the 1970s [24].

The more recent civil war involved the Colombian government and various left-wing guerrilla groups including the Fuerzas Armadas Revolucionarias de Colombia (FARC), National Liberation Army (ELN), and Popular Liberation Army (EPL) [28]. The conflict resulted in millions of individuals displaced from their homes and exceptionally high rates of violence [24]. Although there had been attempts to negotiate peace prior to 2012, until the 2012-2016 negotiations, they were unsuccessful. For example, one negotiation attempt in the 1980s resulted in the creation of a political party (the Unión Patriótica) affiliated with the FARC [28]. However, many of the party's members were assassinated, leading the FARC to separate and continue aggressions [28]. In 2012, a new cycle of peace discussions began between the two groups, which was ultimately agreed upon and ratified in 2016 [28].

Understanding the development and resiliency of peace agreements is vitally important to creating sustainable peace. Recent work shows that support from citizens and civil society actors for peace agreements contributes to their success [27,31]. However, measuring social support for policies or even for various actors in a conflict can be difficult, as the different methods used to measure support can produce different results. For example, individuals asked directly about their preferences in Colombia tend to describe strong support for military groups because of economic and social pressures [28]. Survey data have thus shown high trust in the military over time, but when surveys are designed to ask about preferences indirectly, a much more nuanced picture emerges that shows variations in trust based on an individual's context [28].

Obtaining these more nuanced data are costly, time consuming, logistically challenging, and doing so requires extreme attention to survey details and methodologies [38]. Other data streams, like social media posts and Internet search query patterns, might offer a new mechanism to gauge political sentiment in potentially fraught situations for lower overhead while simultaneously providing access to a larger volume of data [17]. 


\subsection{Data Sources in Political Science}

Historically, political science has relied on traditional sources of data including large surveys (e.g., the census), existing documents (e.g., newspapers, government documents), and primary data (e.g., interview responses, surveys) [29]. One commonly used data source is event-based systems, which automatically categorize events from news media in systematic ways [12]. Recently, the systems have been improved using updated natural language processing techniques, which has resulted in substantially more accurate datasets with higher coverage of global events [12].

One such system is ICEWS [11]. ICEWS is a database of coded events describing contact between various socio-political actors. It gathers data from 100 data sources and 250 international and regional newsfeeds [11]. It was developed with funding from agencies in the U.S. government with the goal of allowing policy analysts to track relevant global events that could require U.S. response (e.g., rebellions, insurgencies) [46].

Beyond traditional sources, many have noted the potential usefulness of novel data sources to gain insight into individuals. Internet data have been used to examine political environments online (via Reddit) [6], identify political trolls [5], and explore the impact of violence on hate speech online [32].

Many in the political science domain have recognized that Internet data are valuable for a number of different insights. Hopke et al. [22] compared Twitter and Facebook users in Colombia and found that those who used Twitter on cell phones were more likely to engage in political conversation.

Separately, others have described the important role that social media played in the Arab Spring [23, 25, 43]. Activists in multiple countries used Twitter to organize country-wide protests and to increase support and visibility for existing annual protests, leading to larger demonstrations [25]. Some, like the Egyptian government, responded by shutting down the Internet for several days, which probably actually increased protest activity [25]. In other cases, like in Tunisia, the Twitter-organized protests eventually led to the resignation of its president, Ben Ali [26].

Importantly, scholars also note that social media access was likely not the cause of the success of protests during the Arab Spring. Rather, social media and Internet capabilities were a component of a much more complex environment that led to the movement [7]. In this context, the Internet is a tool that can be used to organize and is separately a space for people to engage in political debate [7,25].

\subsection{Forecasting Violence in Political Science}

Some headway has been made in forecasting various events of interest in political science. Goldstone et al. [19] were able to forecast the onset of political instability (civil wars, democratic reversals, genocide, and state collapse) with a 2-year lead time with about $80 \%$ accuracy using only a handful of variables grounded in political science literature. The most important variables included a measurement of regime type, infant mortality, discrimination, and neighborhood information (i.e., are neighboring countries experiencing political instability?) [19].

Arva et al. [8] were able to build forecasts with similar accuracy. They compared models built using ICEWS, and a similar but larger dataset, the Global Data on Events, Location and Tone (GDELT), with the goal of forecasting five events of interest: Rebellion, Insurgency, Domestic Crisis, Ethnic or Religious Violence, and International Crisis. Models were of varying quality with recall scores between 0 and 0.86 and precision values between 0 and 0.92 (the model for "insurgency" performed the most poorly) [8].

Hegre et al. [20] focused on making high-resolution forecasts specifically for countries in Africa. They created monthly forecasts for three different types of violence and applied them at the country and subnational level from 2016 to 2021 [20]. Their work uses an ensemble of multiple logistic regression and random forest models to generate predictions. They show that their ensemble model improves on baseline models with an $F 1$ score of $0.74[20]$.

Perhaps most impressive, Ramakrishnan et al. [37] have built a system called EMBERS that ingests data from a wide variety of sources (Twitter, Healthmap, RSS news feeds, satellite data, Google Flu Trends etc.) to forecast 
civil unrest in Latin America. They show that EMBERS can forecast with high recall and precision across a number of countries, though individual metrics vary by country [37].

Finally, there is also work using Internet data for multiple different applications that are more tangentially related. For example, Scanlon and Gerber [39] used topic models derived from posts in online forums as features in regression-based models to forecast the recruitment of violent extremists. They showed that the addition of linguistic information from the topic models produces better forecasts than the time-series-only approaches (e.g., AutoRegressive Moving Average (ARIMA)) [39]. Wu and Gerber [47] focused on forecasting protests in Cairo, Egypt, during the Arab Spring by mapping features from social media data (Twitter) and event-based datasets to protest participation theory to produce predictions of future protest.

The advantage of our work is twofold. First, we explore the addition of a novel data stream: Google Trends. Second, we explore the ability to forecast a specific event, state-based armed conflict, at a higher resolution than most prior work. Like Hegre et al. [20], we use monthly level forecasts compared to prior work that has typically relied on yearly (or coarser) data. In addition, we explicitly measure model staleness, ${ }^{1}$ a component that all prior work, to our knowledge, has not included.

\section{METHODOLOGY}

\subsection{Data Collection}

We used four sources of data for the models presented here:

(1) ICEWS: ICEWS is a database of coded events describing interactions between various socio-political actors with related geographic and temporal metadata from more than 100 data sources and 250 international and regional newsfeeds [11]. Data from Colombia were extracted for 2010-2015. Events are coded based on the Conflict and Mediation Event Observations (CAMEO) taxonomy [40, 41], which characterizes events into codes and subcodes (e.g., in the Top Code Appeal, subcodes include Appeal for material cooperation and Appeal for economic cooperation). The categorization into codes is done using the BBN ACCENT algorithm that varies substantially in accuracy by code [3]. Because top codes tend to have higher precision and recall than subcodes [3], we grouped all events into their top code designation $(N=20)$ for this work. These were further aggregated to monthly resolution to match the other features. In total, there were $N=20$ features from ICEWS.

(2) Google Trends: Monthly popularity of 203 Spanish and English search terms from 2010 to 2015. Search terms were determined by identifying relevant individuals, verbs (e.g., "violence," "kidnap"), and other nouns in consultation with subject matter experts. A Spanish-speaking team member translated our keywords from English to Spanish. Google Trends ${ }^{2}$ provides a normalized estimate of the popularity of a specific term compared to all topics [4]. Very small search volumes are represented as " $\leq 1$." To keep these features continuous, these values were replaced with 0.5 .

(3) Twitter: Monthly prevalence of political relevant tweets. The identification of these tweets is described our prior work [13]. In short, tweets geolocated to Colombia were identified from a large corpus of Twitter data from 2009 to 2019. Topic modeling was used to identify politically relevant topics and tweets were assigned to a topic based on which topic was most prevalent in their distribution. For this work, we included the raw number of tweets per topic and the number of tweets normalized to the volume of tweets in Colombia in our dataset. These features were further stratified by the sentiment of the tweet (e.g., Political topic 1, Positive sentiment and Political topic 2, Neutral sentiment) for a total of $N=10$ features.

\footnotetext{
${ }^{1}$ How often does the model need to be retrained to produce "good" predictions?

${ }^{2}$ https://trends.google.com/trends/.
} 
(4) UCDP Ground Truth: Our ground truth dataset is the armed conflict dataset from the UCDP [18, 35, 45]. These data contain instances of "State-based Armed Conflict." 3 An extensive description of the variables used in this data are provided in the codebook [34].

The final dataset included monthly data from 2010 to 2016 in Colombia. There were a total of 233 features: 20 from ICEWS, 10 from Twitter, and 203 from Google Trends.

\subsection{Model Development}

We used several different models to measure the predictability of violent events. First, we used three different linear models (Ridge, Lasso, and ElasticNet) to see if we could accurately predict how many violent events would occur $n$ month(s) from the end of the training data. These three linear models were selected because they use different regularization parameters. Lasso regression uses the $l_{1}$ penalty, which essentially performs feature selection by reducing the coefficients of some features to zero. Ridge, in contrast, uses the $l_{2}$ penalty to shrink unimportant features' coefficients to be small but non-zero. ElasticNet combines the two and included both types of penalties. The advantage of testing all three linear model types is to assess if a particular type of regularization improves hindcasting capabilities.

In addition, we treated the outcome in a binary fashion: will there be any violent event $n$ month(s) from now? For the binary models we used logistic regression models.

For both model types, we tested model horizons of 1 to 6 months, inclusive, and varied the number of training months. For the linear models, we tested 12 to 48 months of training data, whereas for logistic, we tested 40 to 80 months. The reason for this is because there were more months with violent events than without, especially for the first several years in our dataset. We started with 40 months of training data to give the model a reasonable number of months with no violent events.

Models were parameterized using a 5-fold cross-validation grid search across parameters on the training data. We varied regularization strength in orders of magnitude from 0.0001 to 10,000 and optimization tolerance in orders of magnitude from 0.1 to 0.0001 for all models. Logistic regression models additionally learned the best type of regularization $\left(l_{1}\right.$ or $\left.l_{2}\right)$, and our ElasticNet models varied the $l_{1}$ ratio from 0.1 to 0.9 in steps of 0.1 . All models were implemented using scikit-learn [33].

In addition, we compare our logistic models to a naïve baseline where we simply predict that the outcome in month $n$ is the same as the outcome in the last month of training data (e.g., for a forecast horizon of 1 month, the value would be the outcome in month $n$-1). This method has been used extensively in other literature including that of Scanlon and Gerber [39]. Here, the rationale is that one extremely simple model would be to assume that the future is going to be the same as the recent past.

3.2.1 Feature Sets. For each model, three feature sets were considered:

(1) ICEWS-only: Only data from ICEWS were available.

(2) Internet-only: Only Internet features (Google Trends and Twitter) were available.

(3) Internet + ICEWS: All Internet features (Google Trends and Twitter) and all ICEWS features were available.

In this way, we could evaluate if models built using traditional sources of data (ICEWS) in conjunction with Internet data could outperform those using only Internet or only traditional data.

Because the total number of features was large in comparison to the number of training datapoints available, we first performed feature selection using the scikit-learn implementation of recursive feature elimination and

\footnotetext{
${ }^{3}$ The UCDP program defines this type of conflict as "a contested incompatibility that concerns government and/or territory where the use of armed force between two parties, of which at least one is the government of a state, results in at least 25 battle-related deaths in one calendar year" [16].
} 
cross-validated selection ("RFECV") using five folds [1]. This method uses feature ranking, recursive feature elimination, and cross validation to select the best number of features. A minimum of 10 features were required to be selected, and 1 feature was removed at each step. Eleven features were selected from feature set 1, 184 from feature set 2 , and 39 features were selected from feature set 3 . The features selected and their associated coefficients for each model are shown later in Figure 5 and are discussed in more depth in the following. Interestingly, no Twitter features were selected in any model.

3.2.2 Experiment Design. The experiment was designed to mimic what would be done operationally, where models would be retrained often as new data became available. As such, for each training amount $t$, the data were split into training and testing data such that the first $t$ months were training and the testing data included months $t+1$ to $t+6$ (per the model horizon). The model was then retrained using the data from month 1 to month $t+1$, and tested on months $t+2$ to $t+7$ (et cetera).

3.2.3 Model Metrics. To assess the quality of the models, we use a number of metrics. For linear models, we measure $r^{2}$, root mean squared error (rmse), and hit rate. Hit rate is a measurement of the number of times the forecast correctly predicted an increase or decrease in events. For example, if the model predicted an increase in violent events and that occurred, the model gets a point. The same is true if the model predicted a decrease and that occurred, or predicted that the number of events would stay the same and that occurred. The number of "hits" is divided by the total number of forecasts made.

For logistic models, we use precision, recall, and F1. Precision (also known as the positive predictive value) is the number of true positives divided by the sum of the true positives and false positives. Recall (or sensitivity) is the number of true positives divided by the number classified positive.

\section{RESULTS}

Figure 1 shows some example time series of the data used as features in our models as well as the outcome of interest (violent events). Although it is unclear if there is an immediate pattern with our outcome, there are some interesting temporal trends that reflect the political climate of the time. For example, the Google query 'santos' refers to Juan Manuel Santos who was the president of Colombia during the negotiation of the peace process, and 'calarca' refers to Marcos Calarca who was part of the FARC negotiating team. Searches for 'calarca' increased over time from 2010 to the referendum's ratification in 2016, whereas searches for 'santos' show an increase first in 2010 (when Santos became president) and then show a steady increase in 2014 and 2015 while negotiations were happening.

The linear regression models performed quite poorly (Table 1). The best model was Ridge regression using 48 months of training data and a model horizon of 6 months. This model had an $r^{2}=0.30$, rmse $=9.55$, and hit rate $=0.52$. The best Lasso and ElasticNet performed similarly but with slightly lower $r^{2}$ scores. Note that these are the best models, whereas Table 1 shows the average model and the associated $95 \%$ confidence interval for each metric (i.e., most models perform very poorly, but each model has an outlier that performs slightly less poorly). For this reason, we chose not to discuss the linear regression models further.

The logistic regression models are substantially more promising. Figure 2(a) shows the average $F 1$ score across all models for all three feature sets. The best Internet-only models outperform Internet + ICEWS and ICEWS-only, although only for a handful of forecast horizons (2, 3, and 5 months). Overall, we see that Internet + ICEWS is stable and high performing across all model horizons. Figure 2(b) and (c) show precision and recall, respectively, across all models. Internet + ICEWS models appear to be consistent in both precision and recall, with stronger recall.

As described earlier, precision measures how many of the selected items should have been selected. A precision of 1 means the model identified no false positives, whereas a precision of 0 means the model does not identify any true positive cases, and thus all positive instances are false positives. Recall measures how many of the relevant 
Table 1. Metrics for Linear Models

\begin{tabular}{lccc}
\hline Model & $r^{2}$ & RMSE & Hit Rate \\
\hline Lasso & $0.09(0.08-0.11)$ & $8.07(7.54-8.62)$ & $0.40(0.38-0.42)$ \\
Ridge & $0.09(0.07-0.11)$ & $7.27(6.94-7.61)$ & $0.36(0.35-0.38)$ \\
ElasticNet & $0.09(0.07-0.11)$ & $8.18(7.59-8.78)$ & $0.40(0.38-0.41)$
\end{tabular}

Values shown are averages and $95 \%$ confidence intervals across all training times. All models perform quite poorly, with low $r^{2}$ values and relatively high error.

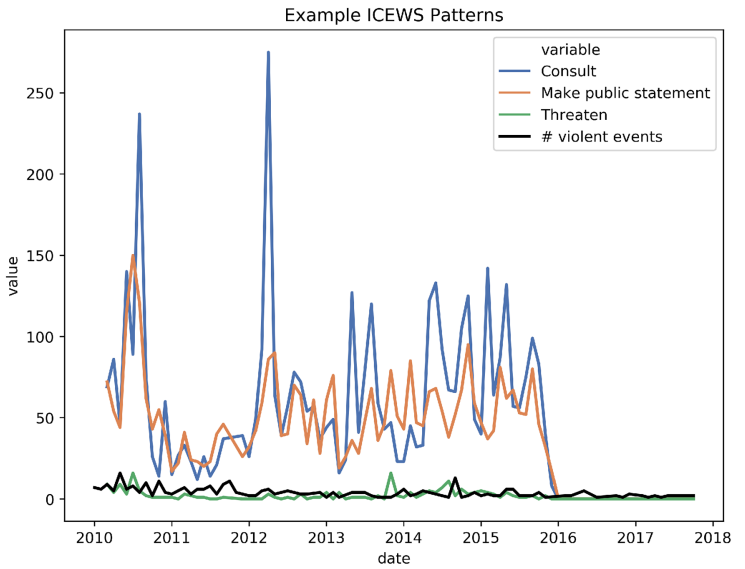

(a) ICEWS

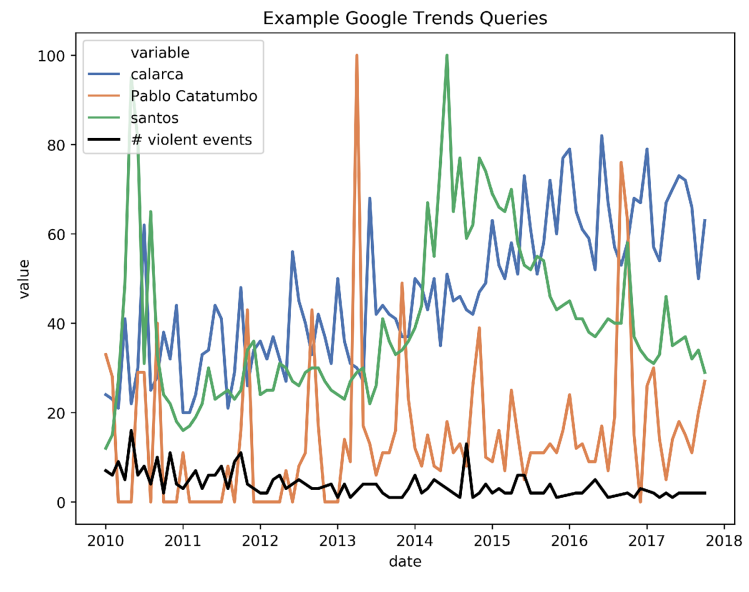

(b) Google search queries

Fig. 1. Example features for Google search queries and ICEWS events. The black line shows the outcome (no. of violent events).

items are selected by the model. A recall of 1 means the model has no false negatives. A recall of 0 means there were no true positives selected.

In the context of these models, our results show that we have higher recall, in general, than precision, which means that we have few false negatives (e.g., when the model predicts no event will happen, it is fairly accurate) but a somewhat higher rate of false positives (e.g., when the Internet + ICEWS model predicts an event will happen at a forecast horizon of 1 month, there is a $70 \%$ chance the model is correct).

The Internet-only models have more variation, both across forecast horizons and within individual models. The ICEWS-only model is also exceptionally stable; however, we do see a somewhat lower recall across most model horizons compared to the Internet + ICEWS model.

The strawman model actually shows the opposite pattern in terms of precision and recall (higher precision than recall), although overall it performs more poorly (i.e., has a lower $F 1$ score) over all forecast horizons. Additionally, even in precision, the best Internet-only models still outperform the best recall models (see Figure 3(b)).

Figure 3 presents the best model (the model with the highest $F 1$ score overall) for each feature set. For Internet + ICEWS, this model was produced using 50 months of training data, at a 1-month forecast horizon. For Internetonly, the best model was from 40 months of training data, and for ICEWS-only, the best model was 43 weeks of training at a 1-month horizon. In all of the best models, recall tends to be higher than precision. Although we did not explicitly optimize for recall, the fact that recall is higher is encouraging. Missing a positive instance, in this domain, is potentially very costly. Said another way, false positives are better than false negatives in this context. 


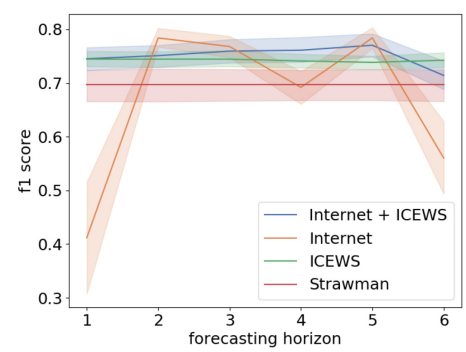

(a) F1

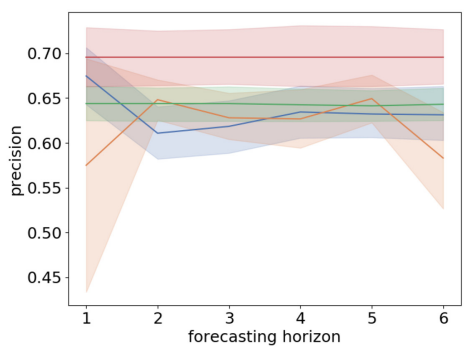

(b) Precision

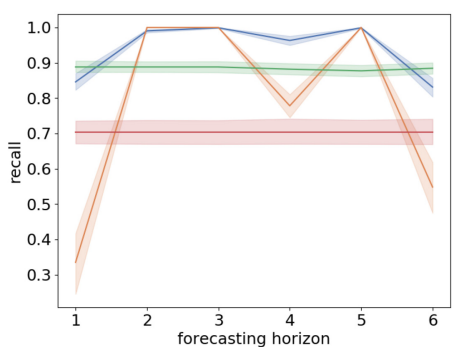

(c) Recall

Fig. 2. Aggregate metrics for models at each forecast horizon. Solid lines show the average value, whereas shaded regions show the $95 \%$ confidence interval.

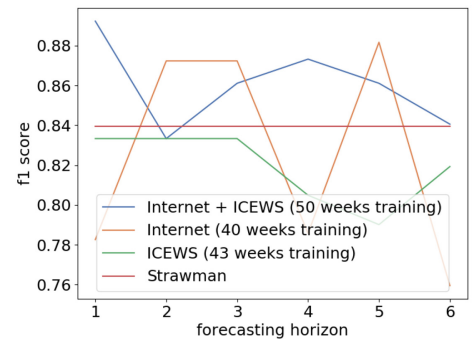

(a) F1

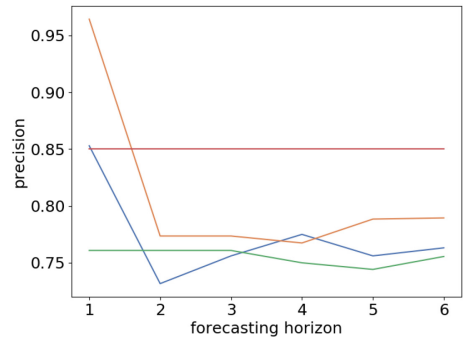

(b) Precision

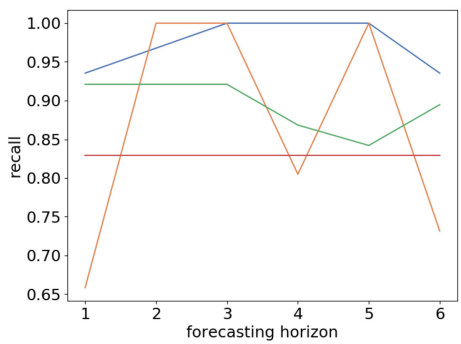

(c) Recall

Fig. 3. Individual metrics for the best model in each feature set at each forecast horizon.

Importantly, a fairly large amount of data are required to produce these models, but too much training data actually produces models with higher variation and lower $F 1$ scores overall (Figure 4).

One reason regression-based models are attractive in this space is because of the interpretability of the model decisions. Figure 5 shows the variables selected in each feature set and the coefficients of each feature based on the amount of training data used. Using this, it is possible to then look at patterns in which coefficients are particularly important. First, we note that despite our initial feature selection step, a large number of the variables included in the final model have weights near 0 , suggesting that the predictive power of most of the variables is extremely limited. Second, variables tend to be similarly weighted regardless of the number of months of training data made available to the models, suggesting that important features remain so regardless of the amount of data available (in our experiments). Third, there are some variables that are routinely weighted more strongly, including features from ICEWS like 'Fight,' 'Threaten,' 'Consult,' and 'Make public statement.' In addition, a number of Google search query terms were somewhat strongly related to our outcome, particularly queries for important public figures like 'Pablo Catumbo,' 'santos,' 'calarca,' and 'de la calle.' Many of the nouns and verbs we included as search query terms (e.g., 'freedom' or 'ilegal' (the Spanish word for illegal)) have non-zero coefficients, but they are largely extremely weak, suggesting that our strongest predictors might be more specific terms related to specific individuals, entities, or events.

\section{DISCUSSION}

In general, we find that we are able to produce high-quality forecasts that outperform our naïve baseline model. Specifically, our results show that the models appear to be resilient to model staleness up to 6 months after the model was initially trained. 


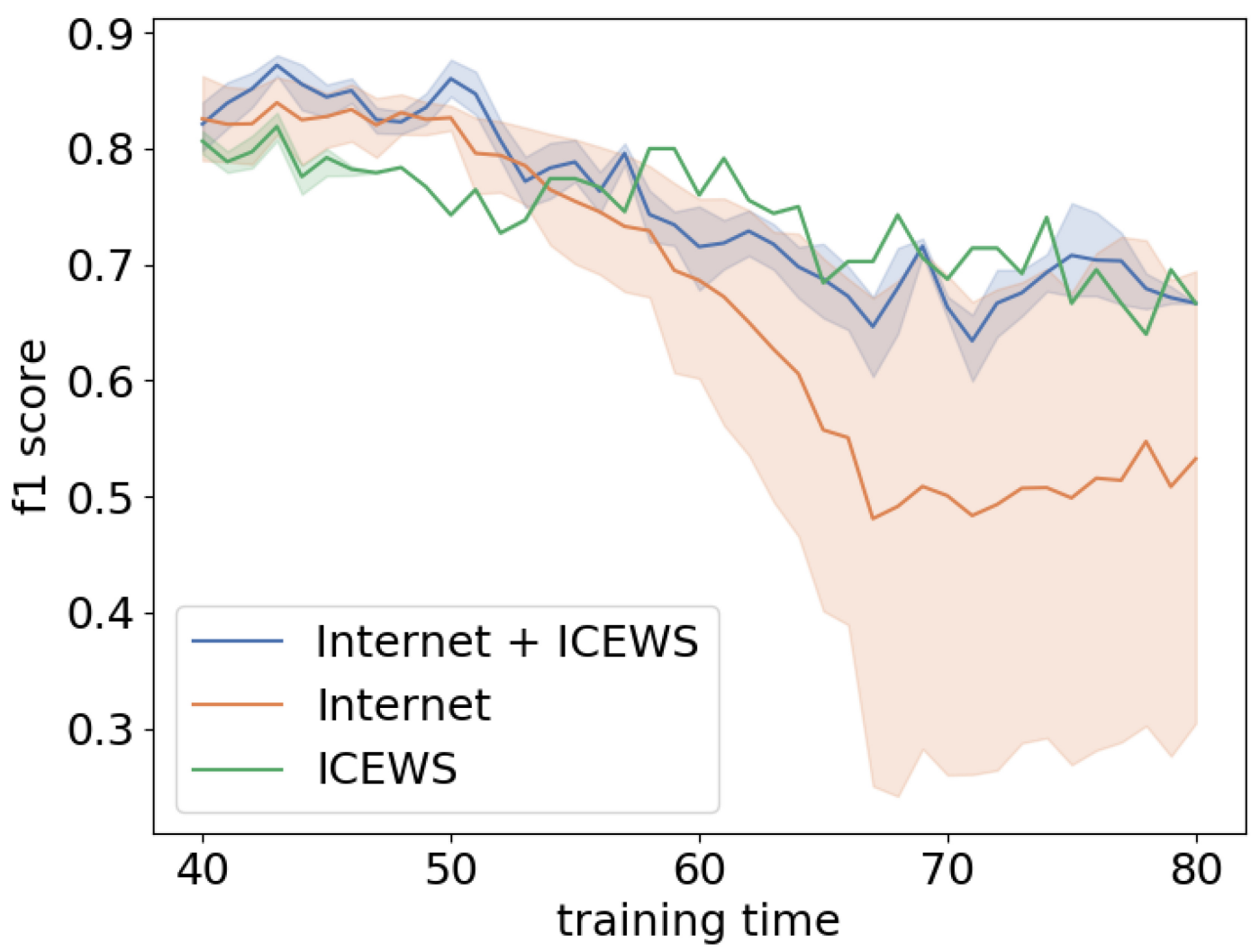

Fig. 4. Impact of training data on F1. Training times are in months. Shaded regions show $95 \%$ confidence intervals.

Second, we observe that although the Internet-only models are reasonably high performing, the addition of a traditional database (ICEWS) produces models that are more consistent, especially at 1- and 6-month forecast horizons. Of note, ICEWS is not the only event-based dataset available. GDELT is a similar but much larger dataset developed through the collaboration of many different academic entities and companies. We chose ICEWS for this work because the ICEWS project has worked on reducing the number of duplicate datapoints and improving sensitivity [8]. Future work might consider the evaluation of other datasets.

Our best models perform quite well $(F 1 \approx 0.80-0.88)$ in both feature sets. Recall stays fairly stable across model horizons, although there is some loss in precision as the horizon increases. The work from Arva et al. [8] bears some similarities. $F 1$ scores for their outcomes of interest varied substantially. Ethnic and religious violence was 0.79 , insurgency was 0 , international crisis was 0.75 , and rebellion (perhaps the outcome most related to our work) was 0.76 . Other similar work from Hegre et al. had $F 1$ scores around 0.74 for country-level predictions of violence in Africa [20]. Our models outperform all of these, and do so at a monthly level, which is a finer temporal resolution than the work from Arva et al. [8].

Although we did not vary the threshold for our logistic regression models because our goal was to produce models with the highest $F 1$ score, it is trivial to do. Such an exercise would vary the tradeoff between precision and recall and could produce a model with a higher precision at the expense of recall. As noted earlier, we believe that our high recall compared to precision could be an asset initially, as the cost of missing an event (a false negative) is potentially more detrimental than forecasting an event that never occurs (false positive); however, there could be contexts where that is not the case (e.g., perhaps the cost of incorrectly predicting an 


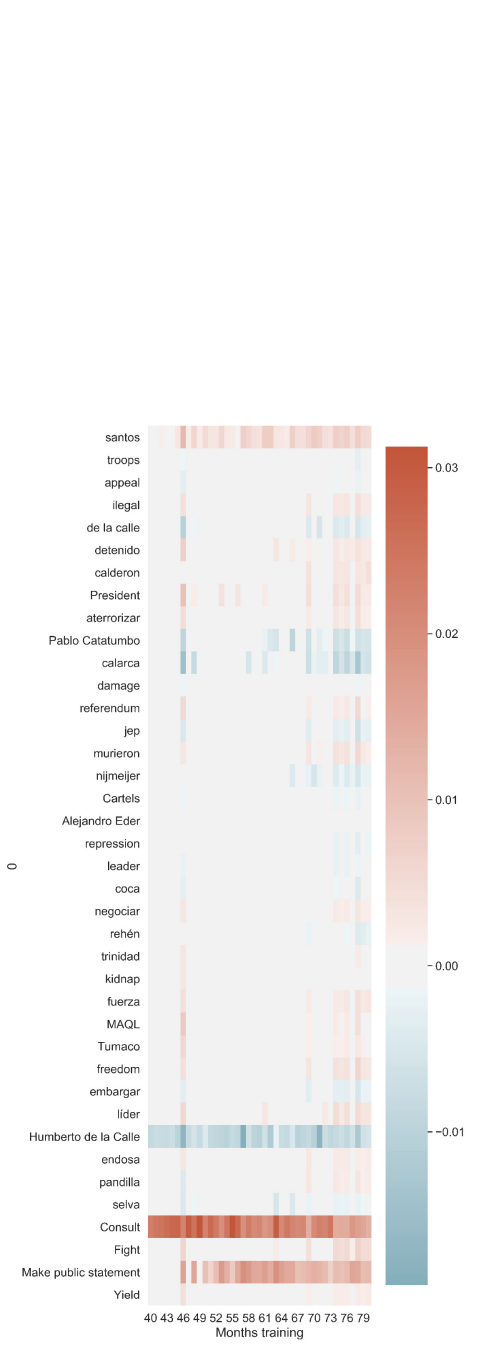

(a) ICEWS + Internet

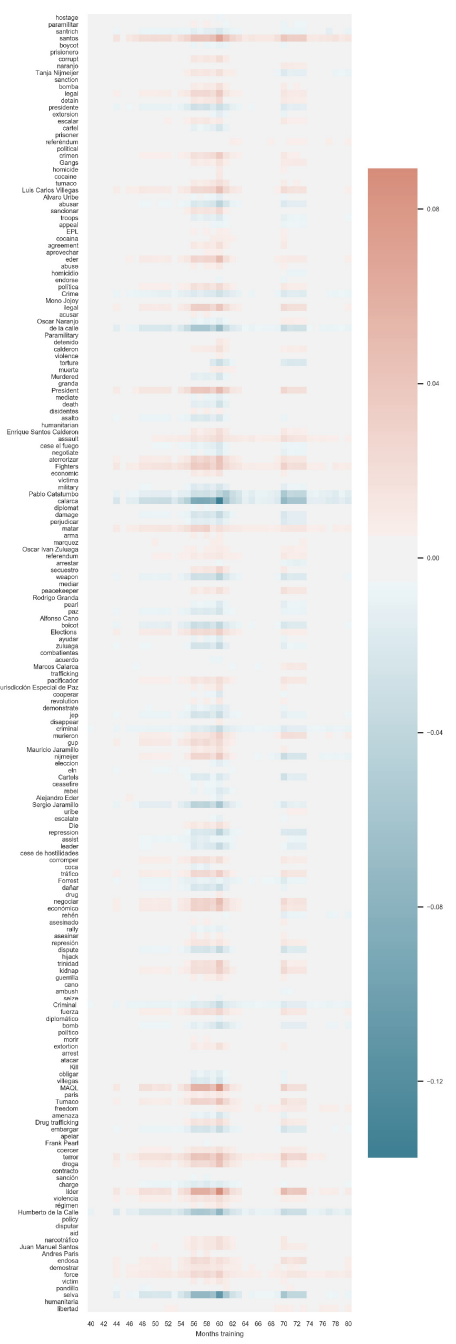

(b) Internet Only

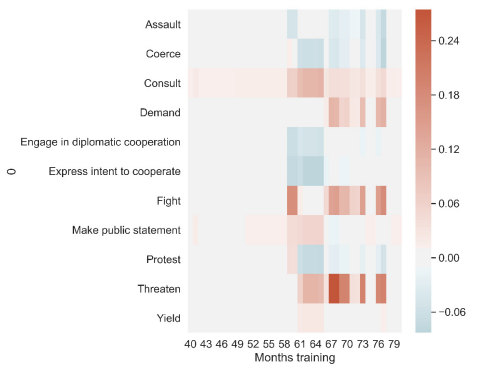

(c) ICEWS Only

Fig. 5. Feature coefficients. Number of months of training data available to the model ( $x$-axis) and features ( $y$-axis) are shown. Color denotes the coefficients (more blue indicates a stronger negative coefficient while more red indicates a more strongly positive coefficient). Even after our initial feature selection step, most of the features have coefficients near 0 .

Digital Government: Research and Practice, Vol. 2, No. 3, Article 28. Publication date: July 2021. 
event is exceptionally high in some situations). Future work might explore this tradeoff to identify models that can function in a number of situations based on various needs.

Additionally, our work finds some interesting patterns in the features most important to our models. First, we find that despite an initial feature selection step, many of the features selected are unimportant and have weights near 0 . Additional work in feature selection could be a useful exercise in future work. In addition, we find that ICEWS events related to negotiations (e.g., 'Consult,' 'Make a public statement') have higher weights in multiple models. This makes sense in the context of the peace negotiations in Colombia, as that is directly related to the ongoing events. However, it is likely that the events of import in other contexts would be dependent on the ongoing political situation specific to that location. In addition, we find that the search query terms that are most important tend to be those related to specific individuals or entities related to the Colombian peace process rather than generic terms. However, we also see that in the Internet-only model, weights are spread out more evenly across a number of terms, including those that are more general.

Interestingly, it is unclear at present what the causal relationship is between observations of search queries and events. It is possible that queries precede events, and also possible that events cause people to search for specific queries. More likely, it is some combination of the two. Understanding this mechanism would allow for more nuanced model development in the future. More generally, political scientist scholars are still debating the benefits of Internet data for political activism. Social media data have garnered most of the attention in this literature, where researchers have noted that most relationships on social media are weak (acquaintances, not strong friends) and argue that this leads to low-risk activities (e.g., "liking" a Facebook post) rather than meaningful political activism [25]. Others point to the Arab Spring protests and describe observed success of using Twitter to organize effective protests $[25,42]$. In general, how search query data might relate to these observed phenomenon and further relate to state-based armed violence is an open question.

\subsection{Limitations}

It is unclear if these findings are generalizable to other contexts. In particular, it would be useful to replicate these experiments in other countries and assess if these findings are consistent at a subnational level. These findings do support the work from Hegre et al. [20] that indicate forecasting of violence in countries in Africa is feasible.

An additional consideration is the possibility that our models are overfitting. In both the ICEWS + Internet and Internet-only models, the number of features selected after the initial feature selection step was large compared to the number of instances available in the training data, although after regularization, many of the coefficients on these variables are quite small. Future work could explore the impact of forcing a stronger regularization parameter or performing different (potentially more aggressive) feature selection prior to model development.

As noted earlier, although we included Twitter features in the initial feature sets, none persisted after feature selection. This could be because our Twitter data initially come from a $1 \%$ random sample of all tweets that our institution has been collecting for several years. Because of the breadth, there is a relatively small number of tweets that can be geolocated to Colombia. Future work would benefit from using additional social media data to assess the usefulness of this particular data stream.

In addition, it bears noting that the usefulness of incorporating Internet features in models relies heavily on their use in different populations, and a good understanding of biases in Internet access and app usage. Although there is some data on demographic biases in Twitter data (e.g., Mislove et al. [30] describe bias in the United States), thorough knowledge about these biases worldwide is lacking. At minimum, various data streams should be investigated for use in a variety of contexts to better understand the benefits and limitations of these features in further models.

Last, as noted, this is not a real-time forecasting work because of the lack of real-time ground truth data. The method presented here is a hindcast rather than a forecast. Evaluating the forecasting capability of the work presented here will need to be done in future studies. 
There are several opportunities for model improvement. First, although regression models provide nice interpretability, other machine learning methods could be investigated if enough data could be amassed. Although we focused on the month time scale, it could be possible to increase the temporal resolution to weekly by using Google Flu Trends data [2]. Note that although the data source explicitly notes the infectious disease "Flu," this dataset includes all searches at a weekly scale for research purposes, not just those related to public health. In addition, it is possible that other methods (e.g., ARIMA or random forests) could be appropriate to explore in future work. Future work could also explore the feasibility of providing subnational forecasts.

\section{CONCLUSION}

We show that a combination of Internet and traditional data sources derived from news media are predictive of violence in Colombia given enough training data. We find models predicting a binary outcome perform better than those predicting a linear outcome. This is likely related to the fact that the binary prediction task is substantially easier than the linear task (i.e., predicting the presence or absence of an event is easier than predicting how many events will occur). Binary outcomes could be forecasted up to 6 months in advance with reasonably high precision and very high recall. Models built using only Internet data features (from Google Trends) perform well, but models built with both data streams are more consistent over a 1- to 6-month model horizon. Internet data, in this case Google search queries, are a promising data source that should be further evaluated for predictive capabilities in this important domain.

\section{ACKNOWLEDGMENTS}

We would like to thank Natalia Garbiras Diaz and Aila Matanock for critical feedback on this project.

\section{REFERENCES}

[1] Feature Ranking with Recursive Feature Elimination and Cross-Validated Selection (RFECV). Retreived on January, 2021 from https: //scikit-learn.org/stable/modules/generated/sklearn.feature_selection.RFECV.html.

[2] Google. Google Flu Trends Data. Accessed November 28, 2020 from https:/www.google.org/flutrends/about/.

[3] Raytheon BBN Technologies. 2015. BBN ACCENT Event Coding Evaluation. Technical Report. Raytheon BBN Technologies.

[4] Google. 2020. FAQ About Google Trends Data. Retrieved May 18, 2021 from https://support.google.com/trends/answer/4365533?hl=en \&ref_topic $=6248052$.

[5] Aseel Addawood, Adam Badawy, Kristina Lerman, and Emilio Ferrara. 2019. Linguistic cues to deception: Identifying political trolls on social media. In Proceedings of the 13th International Conference on Web and Social Media.

[6] Jisun An, Haewoon Kwak, Oliver Posegga, and Andreas Jungherr. 2019. Political discussions in homogeneous and cross-cutting communication spaces. In Proceedings of the 13th International AAAI Conference on Web and Social Media.

[7] Miriyam Aouragh and Anne Alexander. 2011. The Egyptian experience: Sense and nonsense of the Internet revolution. International Journal of Communication 5 (2011), 1344-1358.

[8] Bryan Arva, John Beieler, Bejamin Fisher, Gustavo Lara, Philip A. Schrodt, Wonjun Song, Marsha Sowell, and Sam Stehle. 2013. Improving forecasts of international events of interest. In Proceedings of the EPSA 2013 Annual General Conference. Article 78.

[9] World Bank. 2018. Pathways for Peace: Inclusive Approaches to Preventing Violent Conflict. Technical Report. United Nations, Washington, DC.

[10] Jiang Bian, Umit Topaloglu, and Fan Yu. 2012. Towards large-scale Twitter mining for drug-related adverse events. In Proceedings of the 2012 International Workshop on Smart Health and Wellbeing (SHB'12). ACM, New York, NY, 25. https://doi.org/10.1145/2389707.2389713

[11] Elizabeth Boschee, Jennifer Lautenschlager, Sean O’Brien, Steve Shellman, and James Starz. 2018. ICEWS Automated Daily Event Data. Retrieved May 18, 2021 from https://doi.org/10.7910/DVN/QI2T9A

[12] Elizabeth Boschee, Premkumar Natarajan, and Ralph Weischedel. 2013. Automatic extraction of events from open source text for predictive forecasting. In Handbook of Computational Approaches to Counterterrorism, V. S. Subrahmanian (Ed.). Springer, New York, NY. https://doi.org/10.1007/978-1-4614-5311-6

[13] Ashlynn R. Daughton, Chrysm Watson Ross, Geoffrey Fairchild, and Sara Y. Del Valle. 2019. Topic modeling to contextualize event-based datasets: The Colombian peace process. In Proceedings of the 2nd ACM SIGSPATIAL International Workshop on Advances on Resilient and Intelligent Cities. https://doi.org/10.1145/3356395.3365540

[14] Munmun De Choudhury, Michael Gamon, Scott Counts, and Eric Horvitz. 2013. Predicting depression via social media. In Proceedings of the 7th International AAAI Conference on Weblogs and Social Media.

Digital Government: Research and Practice, Vol. 2, No. 3, Article 28. Publication date: July 2021. 
[15] Munmun De Choudhury, Scott Counts, and Eric Horvitz. 2013. Social media as a measurement tool of depression in populations. In Proceedings of the 5th Annual ACM Web Science Conference (WebSci'13). ACM, New York, NY, 47-56. https://doi.org/10.1145/2464464. 2464480

[16] Department of Peace and Conflict Research. n.d. UCDP Definitions. Retrieved May 18, 2021 from https://www.pcr.uu.se/research/ucdp/ definitions/.

[17] Karsten Donnay. 2017. Big data for monitoring political instability. International Development Policy 8, 1 (Jan. 2017), Article 8.1. https: //doi.org/10.4000/poldev.2468

[18] Nils Petter Gleditsch, Peter Wallensteen, Mikael Eriksson, Margareta Sollenberg, and Håvard Strand. 2002. Armed conflict 1946-2001: A new dataset. Journal of Peace Research 39, 5 (Sept. 2002), 615-637. https://doi.org/10.1177/0022343302039005007

[19] Jack A. Goldstone, Robert H. Bates, David L. Epstein, Ted Robert Gurr, Michael B. Lustik, Monty G. Marshall, Jay Ulfelder, and Mark Woodward. 2010. A global model for forecasting political instability. American fournal of Political Science 54, 1 (Jan. 2010), 190-208. https://doi.org/10.1111/j.1540-5907.2009.00426.x

[20] Håvard Hegre, Marie Allansson, Matthias Basedau, Michael Colaresi, Mihai Croicu, Hanne Fjelde, Frederick Hoyles, et al. 2019. ViEWS: A political violence early-warning system. Journal of Peace Research 56, 2 (March 2019), 155-174. https://doi.org/10.1177/ 0022343319823860

[21] Håvard Hegre, Nils W. Metternich, Håvard Mokleiv Nygård, and Julian Wucherpfennig. 2017. Introduction: Forecasting in peace research. Journal of Peace Research 54, 2 (March 2017), 113-124. https://doi.org/10.1177/0022343317691330

[22] Jill E. Hopke, Itay Gabay, Sojung C. Kim, and Hernando Rojas. 2016. Mobile phones and political participation in Colombia: Mobile Twitter versus mobile Facebook. Communication and the Public 1, 2 (June 2016), 159-173. https://doi.org/10.1177/2057047316642607

[23] Philip N. Howard, Aiden Duffy, Deen Freelon, Muzammil M. Hussain, Will Mari, and Marwa Mazaid. 2011. Opening closed regimes: What was the role of social media during the Arab spring? SSRN Electronic fournal. Retrieved May 18, 2021 from https://papers.ssrn. com/sol3/papers.cfm?abstract_id=2595096.

[24] Ana María Ibáñez and Carlos Eduardo Vélez. 2008. Civil conflict and forced migration: The micro determinants and welfare losses of displacement in Colombia. World Development 36, 4 (April 2008), 659-676. https://doi.org/10.1016/j.worlddev.2007.04.013

[25] Sarah Joseph. 2011. Social media, human rights and political change. SSRN Electronic fournal. Retrieved May 18, 2021 from https:// papers.ssrn.com/sol3/papers.cfm?abstract_id=1856880.

[26] Gilad Lotan, Erhardt Graeff, Mike Ananny, Devin Gaffney, Ian Pearce, and Danah Boyd. 2011. The revolutions were tweeted: Information flows during the 2011 Tunisian and Egyptian revolutions. International fournal of Communication 5 (2011), 1375-1405.

[27] Aila M. Matanock. 2018. How elections can lead to peace: Making negotiated settlements last. Foreign Affairs. Retrieved May 18, 2021 from https://papers.ssrn.com/sol3/papers.cfm?abstract_id=3205656.

[28] Aila M. Matanock and Miguel García-Sánchez. 2017. The Colombian paradox: Peace processes, elite divisions \& popular plebiscites. Daedalus 146, 4 (Oct. 2017), 152-166. https://doi.org/10.1162/DAED_a_00466

[29] David E. McNabb. 2015. Research Methods for Political Science: Quantitative and Qualitative Approaches. Routledge.

[30] Alan Mislove, Sune Lehmann, Yong-Yeol Ahn, Jukka-Pekka Onnela, and J. Niels Rosenquist. 2011. Understanding the demographics of Twitter users. In Proceedings of the 2011 International Conference on Weblogs and Social Media (ICWSM'11).

[31] Desirée Nilsson. 2012. Anchoring the peace: Civil society actors in peace accords and durable peace. International Interactions 38, 2 (April 2012), 243-266. https://doi.org/10.1080/03050629.2012.659139

[32] Alexandra Olteanu, Carlos Castillo, Jeremy Boy, and Kush R. Varshney. 2018. The effect of extremist violence on hateful speech online In Proceedings of the 12th ernational Conference on Weblogs and Social Media (ICWSM'18).

[33] F. Pedregosa, G. Varoquaux, A. Gramfort, V. Michel, B. Thirion, O. Grisel, M. Blondel, et al. 2011. Scikit-learn: Machine learning in Python. Fournal of Machine Learning Research 12 (2011), 2825-2830.

[34] Therése Pettersson. 2019. UCDP/PRIO Armed Conflict Dataset Codebook. Technical Report Version 19.1. Department of Peace and Conflict Research, Uppsala University and Centre for the Study of Civil Wars, International Peace Research Institute, Oslo.

[35] Therése Pettersson, Stina Högbladh, and Magnus Öberg. 2019. Organized violence, 1989-2018 and peace agreements. Journal of Peace Research 56, 4 (July 2019), 589-603. https://doi.org/10.1177/0022343319856046

[36] Reid Priedhorsky, David A. Osthus, Ashlyn R. Daughton, Kelly Moran, Nicholas Generous, Geoffrey Fairchild, Alina Deshpande, and Sara Y. Del Valle. 2017. Measuring global disease with Wikipedia: Success, failure, and a research agenda. In Proceedings of the Conference on Computer-Supported Cooperative Work (CSCW'17).

[37] Naren Ramakrishnan, Patrick Butler, Sathappan Muthiah, Nathan Self, Rupinder Khandpur, Parang Saraf, Wei Wang, et al. 2014. 'Beating the news' with EMBERS: Forecasting civil unrest using open source indicators. arXiv:1402.7035.

[38] Matthew J. Salganik. 2018. Asking questions. In Bit by Bit: Social Research in the Digital Age. Princeton University Press, Princeton, NJ 85-146.

[39] Jacob R. Scanlon and Matthew S. Gerber. 2015. Forecasting violent extremist cyber recruitment. IEEE Transactions on Information Forensics and Security 10, 11 (Nov. 2015), 2461-2470. https://doi.org/10.1109/TIFS.2015.2464775

[40] Philip A. Schrodt. 2013. CAMEO: Conflict and Mediation Event Observations Event and Actor Codebook. Technical Report 1.1b3. Pennsylvania State University. 
[41] Philip A. Schrodt and David Van Brackle. 2013. Automated coding of political event data. In Handbook of Computational Approaches to Counterterrorism, V. S. Subrahmanian (Ed.). Springer, New York, NY, 23-49.

[42] Clay Shirky. 2011. The political power of social media: Technology, the public sphere, and political change. Foreign Affairs 90, 1 (2011), 28-41.

[43] Ekaterina Stepanova. 2011. The role of information communication technologies in the "Arab spring": Implications beyond the region. PONARS Eurasia 15 (May 2011), 1-6.

[44] Yi Tay, Luu Anh Tuan, and Siu Cheung Hui. 2018. COUPLENET: Paying attention to couples with coupled attention for relationship recommendation. In Proceedings of the 12th ernational Conference on Weblogs and Social Media (ICWSM'18).

[45] UCDP. n.d. Uppsala Conflict Data Program. Retrieved May 18, 2021 from https://ucdp.uu.se/.

[46] Michael Ward, Andreas Beger, J. Cutler, M. Dickenson, Cassy Dorff, and Benjamin Radford. 2013. Comparing GDELT and ICEWS event data. Analysis 21 (Jan. 2013), 267-297.

[47] Congyu Wu and Matthew S. Gerber. 2018. Forecasting civil unrest using social media and protest participation theory. IEEE Transactions on Computational Social Systems 5, 1 (March 2018), 82-94. https://doi.org/10.1109/TCSS.2017.2763128

Received April 2020; revised February 2021; accepted April 2021 